Article

\title{
Modular Uniform Convexity of Lebesgue Spaces of Variable Integrability
}

\author{
Mostafa Bachar ${ }^{1}$, Osvaldo Méndez ${ }^{2, *}$ and Messaoud Bounkhel ${ }^{1}$ (D) \\ 1 Department of Mathematics, College of Sciences, King Saud University, Riyadh 11451, Saudi Arabia; \\ mbachar@ksu.edu.sa (M.Bachar); bounkhel@ksu.edu.sa (M.Bounkhel) \\ 2 Department of Mathematical Sciences, University of Texas at El Paso, El Paso, TX 79968, USA \\ * Correspondence: osmendez@utep.edu; Tel.: +1-915-747-6862
}

Received: 12 November 2018; Accepted: 29 November 2018; Published: 3 December 2018

\begin{abstract}
We analyze the modular geometry of the Lebesgue space with variable exponent, $L^{p(\cdot)}$. Our central result is that $L^{p(\cdot)}$ possesses a modular uniform convexity property. Part of the novelty is that the property holds even in the case $\sup p(x)=\infty$. We present specific applications to fixed point theory.
\end{abstract}

Keywords: fixed point theorem; modular uniform convexity; modular vector spaces; Nakano spaces; uniform convexity; variable exponent spaces

\section{Introduction}

In this work, we prove a hitherto unknown modular convexity property of the Lebesgue spaces with variable exponent, $L^{p(\cdot)}$, which has far reaching applications in fixed point theory, remarkably even in the case in which the exponent $p(\cdot)$ is unbounded.

Lebesgue spaces of variable-exponent $\left(L^{p(\cdot)}\right)$ were first mentioned in [1]. In the late $19^{\text {th }}$ century these spaces were brought into the center stage of mathematical research as they were realized to be the natural solution space for partial differential equations exhibiting non-standard growth. The first systematic treatment of variable exponent spaces was given in [2]. In 1997, while studying differential equations in electromagnetism, V. Zhikov's work [3] led to the minimization of integrals of the form

$$
\int_{\Omega}|\nabla w(x)|^{p(x)} d x
$$

which in turn leads to the corresponding Lagrange-Euler equation:

$$
\Delta_{p(\cdot)} w:=\operatorname{div}\left(|\nabla w|^{p(\cdot)-2} \nabla w\right)=0 .
$$

Because of the variability of $p(x)$, Equation (1) is said to have non-standard growth. The natural space for the solutions of such differential equations must take into consideration the dependence of $p(x)$ on the space variable $x$. It is at this point obvious that the classical $L^{p}$ theory is not sufficient in this situation and that a condition such as

$$
\int_{\Omega}|\nabla u(x)|^{p(x)} d x<\infty
$$

should be imposed as an a priori requirement. 
Similar considerations arise in the study of the hydrodynamic equations governing non-Newtonian fluids $[4,5]$. These equations have non-standard growth and model, in particular, electrorheological fluids, i.e., fluids whose viscosity can be changed dramatically and in a few mili-seconds when exposed to a magnetic or an electric field. Electrorheological fluids are currently the object of intense research activity in both theoretical and applied fields. Their applications include medicine, civil engineering and military science [6-9].

Through these applications, then, there inexorably emerged the need for a deeper understanding of these generalized functional spaces with variable integrability.

The article is organized in the following manner: In Section 2 we give the definition of a convex modular and introduce the definition of the UUC2 condition. In Section 3 we lay the ground for our main result by properly defining the Lebesgue spaces with variable integrability. In Section 4, Theorem 3, which constitutes the main contribution of this work, is proved and in Section 5 we present applications.

\section{Modular Spaces}

In the present section we introduce the standard definitions and terminology on modular spaces to be used in the sequel. We also state the concept of modular uniform convexity. For a detailed account of the ideas expounded here, the interested reader is referred to the monograph [10]. Let $V$ be a real or complex vector space. We denote the scalar field with $\mathbb{K}$.

Definition 1. An s-convex modular $(0<s \leq 1)$ on a vector space $V$ over $\mathbb{K}$ is a function

$$
\rho: V \longrightarrow[0, \infty]
$$

that satisfies the following conditions:

1. $\rho(x)=0 \Longleftrightarrow x=0$,

2. $\rho(t x)=|t| \rho(x)$ for any $x \in V,|t|=1$,

3. $\rho(t x+(1-t) y) \leq t^{s} \rho(x)+(1-t)^{s} \rho(y)$ for all $x, y \in V$ and $t \in(0,1]$.

In particular, if $s=1$, the modular is said to be convex. A convex modular $\rho$ on a vector space $V$ is leftcontinuous (right-continuous) if for any $x \in V$ the map

$$
\alpha \longrightarrow \rho(\alpha x)
$$

is left-continuous (right-continuous) on $[0, \infty)$ (or, on $(0, \infty)$ in the case of left continuity); if $\rho$ is both left-and right-continuous, it is said to be continuous.

If $\rho$ satisfies conditions (1) and (2) but not necessarily condition (1), it is said to be a semimodular on $V$. By reason of its relevance to the present work, the following standard example is noted: Consider a domain $\Omega \subseteq \mathbb{R}^{n}$ and set $\mathcal{M}$ to denote the vector space of all Borel-measurable real-valued functions on $\Omega$. Then, the functional

$$
u \longrightarrow \rho_{\infty}(u)= \begin{cases}0, & \text { if } u \text { is bounded a.e., } \\ \infty, & \text { otherwise, }\end{cases}
$$

is a semimodular on $\mathcal{M}$. The following definition is standard [11,12]:

Definition 2. Let $V$ be a real vector space and $\rho$ be a convex modular on $V$. $\rho$ is said to be uniformly convex if for each $\varepsilon>0$ there exists $\delta=\delta(\varepsilon)>0$ such that, for every $u \in V$ and $v \in V$ with $\rho(u)=1, \rho(v)=1$ and $\rho(u-v)>\varepsilon$, it holds that $\rho\left(\frac{u+v}{2}\right)<1-\delta(\varepsilon)$. 
Modular Uniform Convexity

A less stringent form of modular uniform convexity was introduced and studied in [10]. Specifically,

Definition 3. Let $V$ be a real vector space and $\rho$ be a convex modular on $V$. Let $r>0, \varepsilon>0$. Set

$$
D(r, \varepsilon)=\left\{(u, v) \in V \times V: \rho(u) \leq r, \rho(v) \leq r, \rho\left(\frac{u-v}{2}\right) \geq \varepsilon r\right\}
$$

and

$$
\delta(r, \varepsilon)=\inf \left\{1-\frac{1}{r} \rho\left(\frac{u+v}{2}\right):(u, v) \in D(r, \varepsilon)\right\} .
$$

If $D(r, \varepsilon)=\varnothing$, we define $\delta(r, \varepsilon)=1$. Notice that for $\varepsilon>0$ that is small enough, $D(r, \varepsilon) \neq \varnothing$.

Definition 4. The modular $\rho$ is said to be UUC2 (or type 2-uniformly convex, xsee [10]) if for each $s \geq 0$, $\varepsilon>0$, there exists $\eta(s, \varepsilon)>0$ such that for arbitrary $r>s>0$

$$
\delta(r, \varepsilon)>\eta(s, \varepsilon) .
$$

\section{Lebesgue Spaces with Variable Exponent}

In what follows, we delve into the question of uniform convexity of the Lebesgue spaces of variable exponent. We start by stating the basic definitions ([2,13-15]). Given a domain $\Omega \subset \mathbb{R}^{n}, \mathcal{M}(\Omega)$ will stand for the vector space of all real-valued, Borel-measurable functions defined on $\Omega$. We will denote by $\mathcal{P}(\Omega)$ the subset of $\mathcal{M}$ that consists of all functions

$$
p: \Omega \longrightarrow[1, \infty]
$$

As usual, if $A$ is a Borel set $A \subset \mathbb{R}^{n}$, its Lebesgue measure will be written as $|A|$.

Fix such a function $p$, define the sets:

$$
\begin{aligned}
\Omega^{0} & =\{x \in \Omega: 1<p(x)<\infty\}, \\
\Omega^{1} & =\{x \in \Omega: p(x)=1\}, \\
\Omega^{\infty} & =\{x \in \Omega: p(x)=\infty\},
\end{aligned}
$$

and set

$$
p_{-}=\underset{x \in \Omega^{0}}{\operatorname{ess} \inf } p(x) \text { and } p_{+}=\underset{x \in \Omega^{0}}{\operatorname{ess} \sup } p(x) \text { if }\left|\Omega^{0}\right|>0 .
$$

Theorem 1. The function

$$
\begin{aligned}
& \rho_{p}: \mathcal{M}(\Omega) \longrightarrow[0, \infty], \\
& \rho_{p}(u)=\int_{\Omega^{0} \cup \Omega^{1}}|u(x)|^{p(x)} d \mu+\sup _{x \in \Omega^{\infty}}|u(x)|,
\end{aligned}
$$

defines a convex, continuous modular on $\mathcal{M}(\Omega)$.

Proof of Theorem 1. See [2,14].

On the subspace $V$ of $\mathcal{M}(\Omega)$ defined as

$$
V=\left\{v \in V: \exists \lambda>0: \rho_{p}(\lambda v)<\infty\right\},
$$


the functional

$$
\|u\|_{p}=\inf \left\{\lambda>0: \rho_{p}\left(\lambda^{-1} u\right) \leq 1\right\}
$$

is a norm; it is called the Luxemburg norm. Furnished with the Luxemburg norm, $V$ becomes a Banach space. In particular, if the function $p$ is constant, this space coincides with the Lebesgue space $L^{p}$. For this reason, $V$ is called the Lebesgue space of variable exponent or of variable integrability and denoted by $L^{p(\cdot)}(\Omega)$.

To the author's best knowledge, the first reference to the modular given in Theorem 1 is to be found in the work by Orlicz [1]. We refer the reader to $[2,13,14]$ for a systematic treatment of the variable exponent Lebesgue spaces. Notice that if $\left|\Omega^{0}\right|=\left|\Omega^{1}\right|=0$, then $\rho_{p}=\rho_{\infty}$.

We point out in passing that $L^{p(\cdot)}(\Omega)$ is the Musielak-Orlicz space corresponding to the Musielak-Orlicz function

$$
\begin{aligned}
& \varphi: \Omega \times[0, \infty) \longrightarrow[0, \infty) \\
& \varphi(x, t)=t^{p(x)} .
\end{aligned}
$$

These spaces were introduced by Nakano in 1950 [16]; we refer to the surveys [11,13,15] for more detailed information on this vast topic.

If $p$ is constant in $\Omega$, the modular $\rho_{p}$ is simply the $p^{\text {th }}$ power of the Luxemburg norm (5). For this reason, when working whether with the norm or with the modular, one faces essentially the same technicalities. If $p$ is non-constant, however, the situation changes radically. In this case, the handling of the norm presents technical challenges and its often desirable to work with the modular whenever possible. This is especially true when dealing with uniform convexity.

\section{Uniform Convexity}

We recall the following standard definition: a normed space $(X,\|\cdot\|)$ is defined to be uniformly convex iff given any $\varepsilon: 0<\varepsilon \leq 2$ one has

$$
\delta_{X}(\varepsilon)=\inf \left\{1-\left\|\frac{x+y}{2}\right\|:\|x\| \leq 1,\|y\| \leq 1,\|x-y\| \geq \varepsilon\right\}>0 .
$$

The number $\delta_{X}(\varepsilon)$ is known as the modulus of uniform convexity of $X$ (see, for example, $[17,18]$ ). For the variable exponent spaces $L^{p(\cdot)}(\Omega)$, uniform convexity is fully characterized. The reader is referred to $[14,19]$ for the proof of the next Theorem. Notice that it follows that the uniform convexity of the Luxemburg normnin expression (5) is equivalent to the $\Delta_{2}$-condition.

Theorem 2. The following statements are equivalent for any function $p \in \mathcal{P}(\Omega)$ :

(i) $L^{p(\cdot)}(\Omega)$ is uniformly convex;

(ii) $1<p_{-} \leq p_{+}<\infty$;

(iii) The modular $\rho_{p}$ satisfies the $\Delta_{2}$-condition. More precisely, there exists a positive constant $K$ such that for any $v \in L^{p(\cdot)}(\Omega)$ it holds that $\rho_{p}(2 v) \leq K \rho_{p}(v)$.

\section{Modular Uniform Convexity}

Though it follows from Theorem 2 that there is no hope for norm-uniform convexity of $L^{p(\cdot)}(\Omega)$ if the exponent $p$ is unbounded, we will show in this section that even when $p_{+}=\infty$, the modular $\rho_{p}$ still exhibits the uniform-convexity property UUC2 introduced in Definition 3. As will be discussed in Section 6 , this property has far-reaching implications.

To tackle the modular uniform convexity property aim, the following auxiliaries inequalities are necessary:

Lemma 1. Let $a, b, p \in \mathbb{R}$. Then: 
(i) If $p \geq 2$ [17], it holds that

$$
\left|\frac{a+b}{2}\right|^{p}+\left|\frac{a-b}{2}\right|^{p} \leq \frac{1}{2}\left(|a|^{p}+|b|^{p}\right)
$$

(ii) If $1<p \leq 2$ and $|a|+|b| \neq 0$ [20], then

$$
\left|\frac{a+b}{2}\right|^{p}+\frac{p(p-1)}{2}\left|\frac{a-b}{|a|+|b|}\right|^{2-p}\left|\frac{a-b}{2}\right|^{p} \leq \frac{1}{2}\left(|a|^{p}+|b|^{p}\right) .
$$

A detailed proof of (ii) is given in [15].

We next set out to state and prove Theorem 3, which is the central aim of this article.

Theorem 3. Let $\Omega \subseteq \mathbb{R}^{n}$ be open and $p \in \mathcal{P}(\Omega)$. If $\left|\Omega^{\infty}\right|=0$ and $p_{-}>1$ then the modular

$$
\begin{aligned}
& \rho_{p}: L^{p}(\Omega) \longrightarrow[0, \infty), \\
& \rho_{p}(w)=\int_{\Omega}|w(x)|^{p(x)} d x
\end{aligned}
$$

satisfies the UCC2 condition.

Remark 1. The condition $\left|\Omega^{\infty}\right|=0$ cannot be removed, as it is easily shown that $L^{\infty}(\Omega)$ does not have the UUC2 property if $|\Omega|>0$.

Proof of Theorem 3. Fix a domain $\Omega \subseteq \mathbb{R}^{n}$ and $p \in \mathcal{P}(\Omega)$; let $\rho_{p}$ be as in Theorem 1 .

Let $r>0, \varepsilon>0$ and consider $u, v \in D(r, \varepsilon)$, that is, assume that

$$
\rho_{p}(u) \leq r, \rho_{p}(v) \leq r, \rho_{p}\left(\frac{u-v}{2}\right) \geq \varepsilon r .
$$

On account of the convexity of $\rho_{p}$ we have $\varepsilon \leq 1$ : indeed,

$$
r \varepsilon \leq \rho_{p}\left(\frac{u-v}{2}\right) \leq r .
$$

Let $\Omega_{1}:=\{x \in \Omega: p(x) \geq 2\}$. Then, either

$$
\int_{\Omega_{1}}\left|\frac{u(x)-v(x)}{2}\right|^{p(x)} d x \geq \frac{r \varepsilon}{2}
$$

or

$$
\int_{\Omega \backslash \Omega_{1}}\left|\frac{u(x)-v(x)}{2}\right|^{p(x)} d x \geq \frac{r \varepsilon}{2} .
$$

If inequality (6) holds, one has, by virtue of inequality $(i)$ in Lemma 1 :

$$
\begin{array}{r}
\int_{\Omega_{1}}\left|\frac{u(x)-v(x)}{2}\right|^{p(x)} d x+\int_{\Omega_{1}}\left|\frac{u(x)+v(x)}{2}\right|^{p(x)} d x \\
\leq \frac{1}{2}\left(\int_{\Omega_{1}}|u(x)|^{p(x)} d x+\int_{\Omega_{1}}|v(x)|^{p(x)} d x\right) .
\end{array}
$$


It is thus concluded that, in this case,

$$
\int_{\Omega_{1}}\left|\frac{u(x)+v(x)}{2}\right|^{p(x)} d x \leq \frac{1}{2}\left(\int_{\Omega_{1}}|u(x)|^{p(x)} d x+\int_{\Omega_{1}}|v(x)|^{p(x)} d x\right)-\frac{r \varepsilon}{2} .
$$

Thus,

$$
\begin{aligned}
\rho_{p}\left(\frac{u+v}{2}\right)= & \int_{\Omega_{1}}\left|\frac{u(x)+v(x)}{2}\right|^{p(x)} d x+\int_{\Omega \backslash \Omega_{1}}\left|\frac{u(x)+v(x)}{2}\right|^{p(x)} d x \\
\leq & \frac{1}{2}\left(\int_{\Omega_{1}}|u(x)|^{p(x)} d x+\int_{\Omega_{1}}|v(x)|^{p(x)} d x\right)-\frac{r \varepsilon}{2} \\
& +\frac{1}{2}\left(\int_{\Omega \backslash \Omega_{1}}|u(x)|^{p(x)} d x+\int_{\Omega \backslash \Omega_{1}}|v(x)|^{p(x)} d x\right) \\
= & \frac{1}{2}\left(\rho_{p}(u)+\rho_{p}(v)\right)-\frac{r \varepsilon}{2} \\
\leq & r\left(1-\frac{\varepsilon}{2}\right) .
\end{aligned}
$$

On the other hand, if inequality (7) holds, we define

$$
\Omega_{2}:=\left\{x \in \Omega \backslash \Omega_{1}:|u(x)-v(x)| \leq \frac{\varepsilon}{4}(|u(x)|+|v(x)|)\right\} .
$$

With this notation, it follows that

$$
\begin{aligned}
\int_{\Omega_{2}}\left|\frac{u(x)-v(x)}{2}\right|^{p(x)} d x & \leq \frac{\varepsilon}{8}\left(\int_{\Omega_{2}}|u(x)|^{p(x)} d x+\int_{\Omega_{2}}|v(x)|^{p(x)}\right) \\
& \leq \frac{\varepsilon}{8}\left(\rho_{p}(u)+\rho_{p}(u)\right) \leq \frac{r \varepsilon}{4}
\end{aligned}
$$

The validity of statement (7) implies in particular that

$$
\begin{aligned}
\int_{\Omega \backslash\left(\Omega_{1} \cup \Omega_{2}\right)}\left|\frac{u(x)-v(x)}{2}\right|^{p(x)} & =\int_{\Omega \backslash \Omega_{1}}\left|\frac{u(x)-v(x)}{2}\right|^{p(x)}-\int_{\Omega_{2}}\left|\frac{u(x)-v(x)}{2}\right|^{p(x)} \\
& \geq \frac{r \varepsilon}{2}-\frac{r \varepsilon}{4}=\frac{r \varepsilon}{4} .
\end{aligned}
$$

It follows from inequality (ii) in Lemma 1 that, if $x \in \Omega \backslash\left(\Omega_{1} \cup \Omega_{2}\right)$, one has

$$
\begin{aligned}
& \left|\frac{u(x)+v(x)}{2}\right|^{p(x)}+\left(p_{-}-1\right) \frac{\varepsilon}{8}\left|\frac{u(x)-v(x)}{2}\right|^{p(x)} \\
& \leq\left|\frac{u(x)+v(x)}{2}\right|^{p(x)}+\frac{p(x)(p(x)-1)}{2}\left(\frac{\varepsilon}{4}\right)^{2-p(x)}\left|\frac{u(x)-v(x)}{2}\right|^{p(x)} \\
& \leq\left|\frac{u(x)+v(x)}{2}\right|^{p(x)}+\frac{p(x)(p(x)-1)}{2}\left|\frac{u(x)-v(x)}{|u(x)|+|v(x)|}\right|^{2-p(x)}\left|\frac{u(x)-v(x)}{2}\right|^{p(x)} \\
& \leq \frac{1}{2}\left(|u(x)|^{p(x)}+|v(x)|^{p(x)}\right) .
\end{aligned}
$$


Integrating the last inequality over $\Omega \backslash\left(\Omega_{1} \cup \Omega_{2}\right)$, it is easily concluded that

$$
\begin{aligned}
& \int_{\Omega \backslash\left(\Omega_{1} \cup \Omega_{2}\right)}\left|\frac{u(x)+v(x)}{2}\right|^{p(x)} d x+\left(p_{-}-1\right) \frac{\varepsilon}{8} \int_{\Omega \backslash\left(\Omega_{1} \cup \Omega_{2}\right)}\left|\frac{u(x)-v(x)}{2}\right|^{p(x)} d x \\
& \leq \frac{1}{2}\left(\int_{\Omega \backslash\left(\Omega_{1} \cup \Omega_{2}\right)}|u(x)|^{p(x)} d x+\int_{\Omega \backslash\left(\Omega_{1} \cup \Omega_{2}\right)}|v(x)|^{p(x)} d x\right) .
\end{aligned}
$$

We arrive thus at

$$
\begin{aligned}
& \int_{\Omega \backslash\left(\Omega_{1} \cup \Omega_{2}\right)}\left|\frac{u(x)+v(x)}{2}\right|^{p(x)} d x \\
& \leq \frac{1}{2}\left(\int_{\Omega \backslash\left(\Omega_{1} \cup \Omega_{2}\right)}|u(x)|^{p(x)} d x+\int_{\Omega \backslash\left(\Omega_{1} \cup \Omega_{2}\right)}|v(x)|^{p(x)} d x\right)-\left(p_{-}-1\right) \frac{\varepsilon^{2}}{32} r .
\end{aligned}
$$

In all

$$
\begin{aligned}
\rho_{p}\left(\frac{u+v}{2}\right) & =\int_{\Omega_{1} \cup \Omega_{2}}\left|\frac{u(x)+v(x)}{2}\right|^{p(x)} d x+\int_{\Omega \backslash\left(\Omega_{1} \cup \Omega_{2}\right)}\left|\frac{u(x)+v(x)}{2}\right|^{p(x)} d x \\
& \leq \frac{1}{2}\left[\int_{\Omega_{1} \cup \Omega_{2}}|u(x)|^{p(x)} d x+\int_{\Omega_{1} \cup \Omega_{2}}|v(x)|^{p(x)} d x\right] \\
& +\frac{1}{2}\left[\int_{\Omega \backslash\left(\Omega_{1} \cup \Omega_{2}\right)}|u(x)|^{p(x)} d x+\int_{\Omega \backslash \Omega_{1} \cup \Omega_{2}}|v(x)|^{p(x)} d x\right] \\
& -\left(p_{-}-1\right) \frac{\varepsilon^{2}}{32} r \\
& \leq r-\left(p_{-}-1\right) \frac{\varepsilon^{2}}{32} r=r\left(1-\left(p_{-}-1\right) \frac{\varepsilon^{2}}{32}\right) .
\end{aligned}
$$

We conclude that, for any $r>0, \varepsilon>0$ and arbitrary $u, v \in D(r, \varepsilon)$ as specified in Definition 3, it holds that

$$
1-\frac{1}{r} \rho_{p}\left(\frac{u+v}{2}\right) \geq \min \left\{\frac{\varepsilon}{2},\left(p_{-}-1\right) \frac{\varepsilon^{2}}{32}\right\}>0,
$$

and it is concluded by definition that $L^{p(\cdot)}(\Omega)$ is UUC2.

\section{Applications}

A remarkable fact about the above discussion is that the UUC2 property holds even if $p_{+}=$ $\sup _{x \in \Omega} p(x)=\infty$, that is, in the absence of the $\Delta_{2}$ condition. This observation makes the UUC2 condition $x \in \Omega$

a valuable tool for dealing with certain applications that have been hitherto heavily $\Delta_{2}$-dependent. For an exhaustive treatment of the interplay between modular spaces and fixed point theory, we refer the reader to the monograph [10].

Norm convergence is equivalent to modular convergence in $L^{p(\cdot)}(\Omega)$ if and only if $\rho_{p}$ fulfills the $\Delta_{2}$ condition $[13,15]$. Bearing this fact in mind, we introduce some terminology before proceeding any further: a subset $W \in L^{p(\cdot)}(\Omega)$ will be called $\rho_{p}$-bounded if, for some constant $C \geq 0$ and any $u \in W$, the inequality

$$
\rho_{p}(u) \leq C
$$


holds. $W$ is said to be $\rho_{p}$-closed if whenever

$$
u_{n} \stackrel{\rho_{p}}{\rightarrow} u
$$

one has $u \in W$. Notice that, if $p_{+}=\infty$, then $\rho$-closedness and $\rho$-boundedness are strictly weaker than norm-closedness and norm-boundedness, respectively.

The next observation is of particular importance in the sequel. Let $u \in L^{p(\cdot)}(\Omega), v \in L^{p(\cdot)}(\Omega)$ and let $\left(v_{n}\right)$ be $\rho_{p}$-convergent to $v$. Fatou's Lemma yields the following inequality

$$
\rho_{p}(u-v) \leq \liminf _{n \rightarrow \infty} \rho_{p}\left(u-v_{n}\right) .
$$

For obvious reasons, the above is known as the Fatou property of the modular $\rho_{p}$.

Theorem 4. Let $p: \Omega \longrightarrow(1, \infty)$; assume $p_{-}=\inf _{x \in \Omega} p(x)>1$. Let $W \subset L^{p(\cdot)}(\Omega)$ be convex and $\rho_{p^{-c l o s e d}}$ and $u \in L^{p(\cdot)}(\Omega)$ satisfy

$$
d_{\rho_{p}}(u, W)=\inf \left\{\rho_{p}(u-v): v \in W\right\}<\infty .
$$

Then, there exists a unique $v_{0} \in W$ for which

$$
d_{\rho_{p}}(u, W)=\rho_{p}\left(u-v_{0}\right) .
$$

Proof of Theorem 4. One can clearly assume that $u \notin W$, otherwise there is nothing to prove. Under this assumption, one must have $d(u, W)>0$, due to the $\rho_{p}$-closedness of $W$. Let $\left(v_{n}\right) \subseteq W$ be such that

$$
\rho_{p}\left(u-v_{n}\right)<d(u, W)\left(1+\frac{1}{n}\right) .
$$

Then, the sequence $\left(\frac{v_{n}}{2}\right)$ must be $\rho_{p}$-Cauchy, i.e., it must necessarily hold that $\rho_{p}\left(2^{-1}\left(v_{n}-v_{m}\right)\right) \rightarrow 0$ as $m, n \rightarrow \infty$. The latter follows by contradiction. Indeed, if otherwise, there would exist $\delta>0$ and strictly increasing subsequences $\left(n_{k}\right)_{k \geq 1}$ and $\left(m_{k}\right)_{k \geq 1}$ with $n_{k}>m_{k}$ for every $k$ such that

$$
\rho_{p}\left(\frac{v_{n_{k}}-v_{m_{k}}}{2}\right) \geq \delta
$$

for each $k \in \mathbb{N}$. Since $n_{k}>m_{k}$, it would then hold that

$$
\max \left\{\rho_{p}\left(u-v_{n_{k}}\right), \rho_{p}\left(u-v_{m_{k}}\right)\right\} \leq d(u, W)\left(1+\frac{1}{m_{k}}\right):=r_{k} .
$$

Together with the bound (9) and in by virtue of Definitions (3) and (4) and of Theorem 1, there exists $\eta>0$ such that

$$
1-\frac{1}{r_{k}} \rho_{p}\left(u-\frac{\left(v_{m_{k}}+v_{n_{k}}\right)}{2}\right) \geq \eta>0,
$$

for any $k \in \mathbb{N}$. Though not mentioned explicitly there, the proof of Theorem 1 contains the fact that $\eta$ is independent $r_{k}$. Since $W$ is convex by assumption, the last inequality above yields

$$
\begin{aligned}
d(u, W) & \leq \rho_{p}\left(u-\frac{\left(v_{m_{k}}+v_{n_{k}}\right)}{2}\right) \leq r(1-\eta) \\
& =d(u, W)\left(1+\frac{1}{m_{k}}\right)(1-\eta) .
\end{aligned}
$$


Letting $k$ tend to $\infty$ one clearly reaches a contradiction: in conclusion, the sequence $\left(\frac{v_{n}}{2}\right)$ is $\rho_{p}$-Cauchy, as claimed. Since $L^{p(\cdot)}(\Omega)$ is $\rho_{p}$-complete, we define $v$ as

$$
\lim _{n \rightarrow \infty} \rho_{p}\left(v-2^{-1} v_{n}\right)=0 .
$$

Notice that

$$
\rho_{p}\left(2 v-\left(v+\frac{v_{k}}{2}\right)\right) \rightarrow 0 \text { as } k \rightarrow \infty
$$

for fixed $k \in \mathbb{N},\left(\frac{v_{k}+v_{n}}{2}\right)_{n}$ converges to $\frac{v_{k}}{2}+v$. The convexity and $\rho_{p}$-closedness of $W$ imply then that $\frac{v_{k}}{2}+v \in W$ for each $k$ and invoking again the closedness of $W$ we conclude that $2 v \in W$. On account of the Fatou's property for the modular $\rho_{p}$, one concludes that

$$
\begin{aligned}
d(u, W) & \leq \rho_{p}(u-2 v) \leq \liminf _{k \rightarrow \infty} \rho_{p}\left(u-\left(v+\frac{v_{k}}{2}\right)\right) \\
& \leq \liminf _{n \rightarrow \infty} \liminf _{k \rightarrow \infty} \rho_{p}\left(u-\left(\frac{v_{n}+v_{k}}{2}\right)\right) \\
& \leq \liminf _{n \rightarrow \infty} \liminf _{k \rightarrow \infty} \frac{1}{2}\left(\rho_{p}\left(u-v_{n}\right)+\rho_{p}\left(u-v_{k}\right)\right) \\
& =d(u, W) .
\end{aligned}
$$

It follows that

$$
d(u, W)=\rho_{p}(u-2 v)
$$

If $w \in W$ and $d(u, W)=\rho_{p}(u-w)$, it is therefore concluded that

$$
d(u, W) \leq \rho_{p}\left(u-\frac{2 v+w}{2}\right) \leq \frac{1}{2}\left(\rho_{p}(u-2 v)+\rho_{p}(u-w)\right)=d(u, W) .
$$

Since $\rho_{p}$ has the UUC2 property, it is strictly convex. Hence, $w=2 v$, which yields the uniqueness statement.

It should be emphasized at this point that Theorem 4 can be restated as the following minimization result:

Theorem 5. In the notation and under the hypotheses of Theorem 4, there exists a unique solution $u_{0} \in W$ to the minimization problem

$$
\inf _{u-w \in W} \int_{\Omega}|w(x)|^{p(x)} d x
$$

(notice here that $u \in L^{p(\cdot)}(\Omega)$ ).

Proof of Theorem 5. It is immediate from Theorem 4 that the unique solution is given by $w=u-v_{0}$.

Aiming at presenting further applications of the $U U C 2$ property for $L^{p(\cdot)}(\Omega)$, we state and prove Theorem 6:

Theorem 6. Consider a non-increasing sequence $\left(C_{n}\right)_{n}$ of $\rho_{p}$-closed, convex, nonempty subsets of $L^{p(\cdot)}(\Omega)$ and assume that

$$
p_{-}>1
$$


Suppose that for some $v \in L^{p(\cdot)}(\Omega)$ it holds that $\sup d\left(v, C_{n}\right)<\infty$. Then,

$$
\bigcap_{n=1}^{n \geq 1} C_{n} \neq \varnothing \text {. }
$$

Proof of Theorem 6. It is sufficient to assume that, for some $n_{0} \in \mathbb{N}$, it holds that $v \notin C_{n_{0}}$; otherwise there would be nothing to prove. From the $\rho_{p}$-closedness of $C_{n_{0}}$, it is easily derived that $d\left(v, C_{n_{0}}\right)>0$. Since the sequence $\left(C_{n}\right)_{n}$ is non-increasing by assumption, the inequalities

$$
\infty>\sup _{n \geq 1} d\left(v, C_{n}\right) \geq d\left(v, C_{n}\right)=\inf _{u \in C_{n}} d(v, u) \geq \inf _{u \in C_{n-1}} d(v, u)=d\left(v, C_{n-1}\right)
$$

are clear for any $n>1$. Thus, the sequence $d\left(v, C_{n}\right)$ is non-decreasing and bounded. Let $L=$ $\lim _{n \rightarrow \infty} d\left(v, C_{n}\right)<\infty$; clearly $L>0$. For each $n \in \mathbb{N}$, let $u_{n} \in C_{n}$ be chosen so that $\rho_{p}\left(v-u_{n}\right)=$ $d\left(v, C_{n}\right)$. As in Theorem 4 , one can prove that the sequence $\left(\frac{u_{n}}{2}\right)_{n}$ is $\rho_{p}$-Cauchy in $L^{p(\cdot)}(\Omega)$ and hence it $\rho_{p}$-converges to, say, $u / 2 \in L^{p(\cdot)}(\Omega)$. Fix $k \in \mathbb{N}$. Then, the sequence $\left(\frac{u_{n}}{2}\right)_{n \geq k}$ is contained in $C_{k}$ and $\rho_{p}$-converges to $\frac{u}{2}$, which implies that $\frac{u}{2} \in C_{k}$, since $C_{k}$ is $\rho_{p}$-closed. In conclusion,

$$
\frac{u}{2} \in \bigcap_{n=1}^{\infty} C_{n}
$$

i.e., $\bigcap_{n=1}^{\infty} C_{n} \neq \varnothing$, as claimed.

To facilitate the proof of the following theorem, we recall the following:

Definition 5. A family $\left(C_{i}\right)_{i \in I}$ of sets is said to have the finite intersection property if for every finite subset $\left\{i_{1}, \ldots i_{k}\right\} \subset I$ it holds that $\bigcap_{j=1}^{k} C_{i_{j}} \neq \varnothing$.

Theorem 7. Assume that $p_{-}>1$ and suppose that $\varnothing \neq C \subset L^{p(\cdot)}(\Omega)$ is a $\rho_{p}$-closed, $\rho_{p}$-bounded, convex set, then if let $\left(C_{i}\right)_{i \in I} \subset 2^{C}$ is a family of subsets of $C$ having the finite intersection property, it necessarily holds that

$$
\bigcap_{i \in I} C_{i} \neq \varnothing
$$

Proof of Theorem 7. $C$ is $\rho_{p}$-bounded; it is therefore immediate that, for any $u \in C$ and $i \in I$,

$$
d\left(u, C_{i}\right)=\inf _{v \in C_{i}} \rho_{p}(u-v) \leq \sup _{v \in C} \rho_{p}(u-v)<\infty .
$$

For any finite subset $A \subset I$, let

$$
d_{A}=d\left(u, \bigcap_{j \in A} C_{j}\right)
$$

Notice that if $A$ and $B$ are finite subsets of $I$ and if $A \subseteq B$, then $\bigcap_{j \in B} C_{j} \subseteq \bigcap_{j \in A} C_{j}$. Consequently,

$$
d\left(u, \bigcap_{j \in A} C_{j}\right)=\inf _{v \in \cap_{j \in A} C_{j}} \rho_{p}(u, v) \leq \inf _{v \in \cap_{j \in B} C_{j}} \rho_{p}(u, v),
$$


i.e., $d_{A} \leq d_{B}$. Write

$$
d_{I}=\sup \left\{d\left(u, \bigcap_{i \in J} C_{i}\right) J \subset I: \text { and } \bigcap_{i \in J} C_{i} \neq \varnothing\right\} .
$$

Let $\left(A_{n}\right)$ be the sequence defined by

$$
d_{I}-\frac{1}{n}<d_{A_{n}} \leq d_{I}
$$

Write $B_{n}=\bigcup_{k=1}^{n} A_{k}$ and $J=\bigcup_{n=1}^{\infty} B_{n}$. It is clear then that, for each $n \in \mathbb{N}$, the set $\bigcap_{i \in B_{n}} C_{i}$ is $\rho_{p}$-closed, convex and non-empty and that the sequence $\left(\bigcap_{i \in B_{n}} C_{i}\right)$ is non-increasing. Hence, Theorem 6 applies and we have

$$
S=\bigcap_{i \in J} C_{i} \neq \varnothing .
$$

By definition, for each $n \in \mathbb{N}$, it holds that

$$
\bigcap_{i \in J} C_{i} \subseteq \bigcap_{i \in A_{n}} C_{i}
$$

and it follows that for each $n$ one has

$$
d_{I}-\frac{1}{n}<d_{A_{n}} \leq d(u, S) \leq d_{I}
$$

Thus, $d(u, S)=d_{I}$. On account of Theorem 4 , there exists a unique $z \in S$ which satisfies $\rho_{p}(u-z)=d_{I}$ and, therefore, for any index $i_{0} \in I$, one has

$$
S \supseteq S \cap C_{i_{0}}=\bigcap_{i \in J \cup\left\{i_{0}\right\}} C_{i} \neq \varnothing ;
$$

it is seen immediately that $d_{I} \leq d(u, S) \leq d\left(u, S \cap C_{i_{0}}\right) \leq d_{I}$. In all,

$$
d(u, S)=d\left(u, S \cap C_{i_{0}}\right)
$$

and by Theorem 4 there exists a unique $w \in S \cap C_{i_{0}}$ for which

$$
\rho_{p}(u-w)=d\left(u, S \cap C_{i_{0}}\right)=d_{I} .
$$

In particular, $w \in S$, thus, invoking the uniqueness part of Theorem 4 , one must necessarily have $w=z$. Since $i_{0}$ is arbitrary, it is concluded that $z \in \bigcap_{i \in I} C_{i}$ and hence the latter intersection is non-empty, as claimed.

The following theorem is another consequence of the UUC2 property for $L^{p(\cdot)}(\Omega)$.

Theorem 8. Let $p_{-}>1, \varnothing \neq C \subset L^{p(\cdot)}(\Omega)$ be a convex, $\rho_{p}$-closed, $\rho_{p}$ bounded and assume that $C$ is not a singleton (i.e., $C$ at least two distinct points). Then, there exists $u \in C$ for which

$$
\sup _{v \in C} \rho_{p}(u-v)<\operatorname{diam}(C)
$$

where as usual $\operatorname{diam}(C)=\sup _{a, b \in C} \rho_{p}(a-b)$ stands for the $\rho_{p}$-diameter of $C$. 
The property established in Theorem 8 is commonly referred to as the $\rho_{p}$-normal structure property. Theorem 8 can thus be rephrased as asserting that, if $p_{-}>1$, then $L^{p(\cdot)}(\Omega)$ has $\rho_{p}$-norma structure.

Proof of Theorem 8. The assumptions imply that $\delta(C)>0$ and that there exist two distinct points $u \in C, v \in C, u \neq v$. For any $w \in C$, invoking the $U U C 2$ property, it follows at once that, for $\delta$ as in the definition of UUC2, (Definition (3)),

$$
\begin{aligned}
\rho_{p}\left(\frac{u+v}{2}-w\right) & =\rho_{p}\left(\frac{u-w+v-w}{2}\right) \\
& \leq \operatorname{diam}(C)\left(1-\delta\left(\operatorname{diam}(C), \frac{\varepsilon}{\operatorname{diam}(C)}\right)\right) .
\end{aligned}
$$

The arbitrariness of $w$ in concert with the convexity of $C$ yields the claim.

Theorem 9. If $p_{-}>1$ and $\varnothing \neq C \subset L^{p(\cdot)}(\Omega)$ is convex, $\rho_{p^{-}}$-closed and $\rho_{p}$-bounded, it follows that any map

$$
T: C \longrightarrow C
$$

for which the bound

$$
\rho_{p}(T(u)-T(v)) \leq \rho_{p}(u-v)
$$

holds for any $u \in C, v \in C$, has a fixed point. In other words, under the above conditions, there exists $w \in C$ such that

$$
T(w)=w .
$$

Proof of Theorem 9. It is obvious that the theorem is true if $C$ is a singleton. Thus, it can be assumed that the cardinality of $C$ is at least 2 . Let

$$
\mathcal{F}=\left\{\varnothing \neq K \subset C: K \text { is } \rho_{p} \text {-closed and } T(K) \subseteq K\right\} .
$$

Since $C \in \mathcal{F}, \mathcal{F} \neq \varnothing$. Moreover, $\mathcal{F}$ is partially ordered by the order relation

$$
X \leq Y \Longleftrightarrow Y \subseteq X .
$$

If $\mathcal{G}$ is a totally order subfamily of $\mathcal{F}$, then $\mathcal{G}$ possesses the finite intersection property and, on account of Theorem 7, it follows that

$$
\bigcap_{X \in \mathcal{G}} X \neq \varnothing ;
$$

this clearly implies that $\bigcap_{X \in \mathcal{G}} X \in \mathcal{F}$, hence $\bigcap_{X \in \mathcal{G}} X$ is an upper bound for $\mathcal{G}$.

Zorn's Lemma yields the existence of a maximal element $X_{0} \in \mathcal{F}$. We set about to prove that $X_{0}$ contains exactly one point. Denote the intersection of all $\rho_{p}$-closed, convex subsets of $C$ that contain $T\left(X_{0}\right)$ by $\overline{\operatorname{conv}}^{\rho_{p}}\left(T\left(X_{0}\right)\right)$. In particular, since $X_{0} \in \mathcal{F}$,

$$
\overline{\operatorname{conv}}^{\rho} p\left(T\left(X_{0}\right)\right) \subseteq X_{0} .
$$

On the other hand, the set $\overline{\operatorname{conv}} \rho_{p}\left(T\left(X_{0}\right)\right)$ belongs to $\mathcal{F}$ because it is convex, $\rho_{p}$-closed and it holds that

$$
T\left(\overline{\operatorname{conv}}^{\rho_{p}}\left(T\left(X_{0}\right)\right)\right) \subseteq T\left(X_{0}\right) \subseteq \overline{\operatorname{conv}}^{\rho_{p}}\left(T\left(X_{0}\right)\right) .
$$

As a consequence of the maximality of $X_{0}$ with respect to the indicated inclusion, one has

$$
{\overline{\text { conv }^{\rho}}}_{p}\left(T\left(X_{0}\right)\right)=X_{0} .
$$


Theorem 8 yields the existence of an element $x_{0} \in X_{0}$ such that

$$
r_{0}=\sup _{u \in X_{0}} \rho_{p}\left(x_{0}-u\right)<\operatorname{diam}\left(X_{0}\right) .
$$

Let $B_{\rho_{p}}(a, s)$ denote the $\rho_{p}$-ball of radius $s$ centered at $a$; we remark the obvious fact that the convexity and the Fatou property of the modular $\rho_{p}$ imply that $B_{\rho_{p}}(a, s)$ is $\rho_{p}$-closed and convex. Set

$$
M=\bigcap_{v \in X_{0}} B_{\rho_{p}}\left(v, r_{0}\right) \cap X_{0}=\left\{u \in X_{0}: \sup _{v \in X_{0}} \rho_{p}(u-v) \leq r_{0}\right\} ;
$$

then, $M$ is $\rho_{p}$-closed and convex and $M \subset X_{0}$. Moreover, if $x \in M$, then for any $v \in X_{0}$

$$
\rho_{p}(T(x)-T(v)) \leq \rho_{p}(x-v) \leq r_{0} .
$$

In other words, if $v \in X_{0}, \rho_{p}(T(v)-T(x)) \leq r_{0}$, i.e., $T\left(X_{0}\right) \subseteq B_{\rho_{p}}\left(T(x), r_{0}\right)$. By definition of $\overline{\text { conv }}^{\rho_{p}}\left(T\left(X_{0}\right)\right)$, it is plain that:

$$
\overline{\operatorname{conv}}^{\rho_{p}}\left(T\left(X_{0}\right)\right) \subseteq B_{\rho_{p}}\left(T(x), r_{0}\right) ;
$$

from equality (11), it follows that

$$
X_{0}=\overline{\operatorname{conv}}^{\rho_{p}}\left(T\left(X_{0}\right)\right) \subseteq B_{\rho_{p}}\left(T(x), r_{0}\right) ;
$$

that is, for any $v \in X_{0}, \rho_{p}(T(x)-v) \leq r_{0}$, i.e., $T(x) \in B_{\rho_{p}}\left(v, r_{0}\right)$. It is clear that, by definition of $M$,

$$
T(M) \subseteq M,
$$

so that $M \in \mathcal{F}$ and, since $M \subseteq X_{0}$ and $X_{0}$ is maximal, one has a fortiori:

$$
X_{0}=M
$$

By definition, then, if $w \in X_{0}$,

$$
\rho_{p}\left(w-x_{0}\right) \leq r_{0} ;
$$

this forces the inequality $\operatorname{diam}\left(X_{0}\right) \leq r_{0}$, which contradicts the strict inequality (12) unless $\operatorname{diam}\left(X_{0}\right)=$ 0 . Hence, $\operatorname{diam}\left(X_{0}\right)=0$ and $X_{0}=\{a\}$ is a singleton. Since also $T\left(X_{0}\right) \subseteq X_{0}$, necessarily

$$
T(a)=a .
$$

In conclusion, $T$ has a fixed point.

\section{Conclusions}

The main results in this work can be summarized as follows: Theorem 3 asserts that, if $p_{-}>1$, then the variable exponent space $L^{p(\cdot)}(\Omega)$ has the UUC2 property.

It follows from Theorem 7 that, if $p_{-}>1$ and $C \subset L^{p(\cdot)}(\Omega)$ is a nonempty, $\rho_{p}$-closed, $\rho_{p}$-bounded, convex set, then any family of subsets of $C$ that has the finite intersection property has nonempty intersection.

In Theorem 9, it is proved that, if $p_{-}>1$, then any non-expansive map $T$ on a nonempty, $\rho_{p}$-closed, $\rho_{p}$-bounded, convex subset of $L^{p(\cdot)}(\Omega)$ has a fixed point.

Author Contributions: All authors contributed equally to this article.

Funding: This research was funded by Deanship of Scientific Research at King Saud University, Grant No. RG-1435-079. 
Acknowledgments: The authors would like to express their deep appreciation to the Deanship of Scientific Research at King Saud University for supporting this Research group No. (RG-1435-079). The authors profusely thank the referees for their valuable comments.

Conflicts of Interest: The authors declare no conflict of interest.

\section{References}

1. Orlicz, W. Über konjugierte Exponentenfolgen. Stud. Math. 1931, 3, 200-211. [CrossRef]

2. Kováčik, O.; Rákosník, J. On spaces $L^{p(x)}, W^{k, p(x)}$. Czechoslov. Math. J. 1991, 41, 592-618.

3. Zhikov, V.V. On some variational problems. Rus. J. Math. Phys. 1997, 5, 105-116.

4. Rajagopal, K.; Ruzicka, M. On the modeling of electrorheological materials. Mech. Res. Commun. 1996, 23, s401-s407. [CrossRef]

5. Ruzicka, M. Electrorheological Fluids: Modeling and Mathematical Theory; Lecture Notes in Mathematics 1748; Springer: Berlin, Germany, 2000.

6. Bansevicius, R.; Virbalis, J.A. Two-dimensional Braille readers based on electrorheological fluid valves controlled by electric field. Mechatronics 2007, 17, 570-577. [CrossRef]

7. Chen, J.Z.; Liao, W.H. Design, testing and control of a magnetorheological actuator for assistive knee braces. Smart Mater. Struct. 2010, 19, 035029. [CrossRef]

8. Choi, S.H.; Kim, S.; Kim, P.; Park, J.; Choi, S.B. A new visual feedback-based magnetorheological haptic master for robot-assisted minimally invasive surgery. Smart Mater. Struct. 2015, 24, 065015. [CrossRef]

9. Spencer, B.; Yang, G.; Carlson, J.; Sain, M. “Smart” Dampers for Seismic Protection of Structures: A Full-Scale Study. In Proceedings of the Second World Conference on Structural Control, Kyoto, Japan, 28 June-1 July 1998.

10. Khamsi, M.A.; Kozlowski, W. Fixed Point Theory in Modular Function Spaces; Birkäuser: Basel, Switzerland, 2015.

11. Musielak, J. Orlicz Spaces and Modular Spaces; Lecture Notes in Mathematics, No. 1034; Springer: Berlin/Heidelberg, Germany, 1983.

12. Nakano, H. Topology of Linear Topological Spaces; Maruzen: Tokyo, Japan, 1951.

13. Diening, L.; Harjulehto, P.; Hästö, P.; Růžička, M. Lebesgue and Sobolev Spaces with Variable Exponents; Lecture Notes in Mathematics; Springer: Heidelberg, Germany, 2011; Volume 2017.

14. Edmunds, D.E.; Lang, J.; Méndez, O. Differential Operators on Spaces of Variable Integrability; World Scientific: Singapore, 2014.

15. Méndez, O.; Lang, J. Analysis on Function Spaces of Musielak-Orlicz Type; Taylor and Francis: Didcot, UK, 2018; in press.

16. Nakano, H. Modulared Semi-Ordered Linear Spaces; Maruzen: Tokyo, Japan, 1950.

17. Clarkson, J. Uniformly Convex Spaces. Trans. Am. Math. Soc. 1936, 40, 396-414. [CrossRef]

18. James, R. Uniformly non-square Banach spaces. Ann. Math. 1964, 80, 542-550. [CrossRef]

19. Lukeš, J.; Pick, L.; Pokorný, D. On geometric properties of the spaces $L^{p(x)}(\Omega)$. Rev. Mat. Complut. 2011, 24, 115-130. [CrossRef]

20. Sundaresan, K. Uniform convexity of Banach spaces $\ell\left(\left\{p_{i}\right\}\right)$. Stud. Math. 1971, 39, 227-231. [CrossRef]

(C) 2018 by the authors. Licensee MDPI, Basel, Switzerland. This article is an open access article distributed under the terms and conditions of the Creative Commons Attribution (CC BY) license (http:/ / creativecommons.org/licenses/by/4.0/). 